\title{
ESTRESSE OCUPACIONAL EM TRABALHADORES DE UMA AUTOESCOLA DE JANAÚBA, MINAS GERAIS
}

\section{OCCUPATIONAL STRESS IN WORKERS OF A DRIVING SCHOOL IN JANAÚBA, MINAS GERAIS}

\author{
Ernandes Gonçalves Dias' \\ Maria Luiza Silva Faria ${ }^{2}$
}

\begin{abstract}
Resumo: Objetivou-se traçar o perfil sociodemográfico, histórico ocupacional e identificar os principais fatores estressores entre os colaboradores de uma Autoescola em Janaúba, Minas Gerais. Trata-se de um estudo descritivo de natureza quantitativa, realizado com 18 funcionários de uma autoescola. Os dados foram coletados no período de maio a junho de 2016, por meio de um questionário estruturado em três dimensões (I: dados socioeconômicos; II: informações ocupacionais; III: informações específicas causadoras de estresse). Os resultados mostraram que $72 \%$ dos profissionais estudados eram do sexo masculino. A idade variou de 25 a 45 anos e o estado civil entre casados e solteiros, sendo que $78 \%$ têm como escolaridade máxima o $2^{\circ}$ grau completo. Observou-se que os principais e mais frequentes fatores desencadeadores do estresse ocupacional foram o excessivo controle, por parte da gerência, nas atividades desempenhadas, responsabilidade não claramente definida e falta de reconhecimento pelo trabalho realizado. Acredita-se que esses fatores podem justificar que os trabalhadores entendam que suas sugestões não são consideradas pelos superiores, na tomada de decisões, assim como podem influenciar na percepção de que o trabalho do colega de serviço seja insatisfatório e gerar a sensação de falta de cumprimento da ética profissional e impressão de deslealdade por parte dos colegas. Dessa forma, é importante a valorização dos profissionais de modo a proporcionar excelência em seus serviços prestados, destacando, ainda, os benefícios de um ambiente favorável à boa rotina de trabalho e, posteriormente, à qualidade de vida dos trabalhadores.
\end{abstract}

Palavras-chave: Saúde do trabalhador; esgotamento profissional; fadiga.

Abstract: The objective was to outline the sociodemographic profile, occupational history and to identify the main stressors among the employees of a driving school in Janaúba, Minas Gerais. This is a descriptive study of quantitative nature, conducted with 18 employees of a driving school. Data were collected from May to June 2016 by means of a questionnaire structured in three dimensions (I: socioeconomic data, II: occupational information, III: specific information causing stress). The results showed that $72 \%$ of the professionals studied were male. The age ranged from 25 to 45 years old and the marital status between married and unmarried, with $78 \%$ presenting as maximum schooling the completed high school. It was observed that the main and most frequent factors triggering occupational stress were the excessive control by the management in the activities performed, responsibility not clearly defined and lack of recognition for the work done. It is believed that these factors may justify workers' understanding that their suggestions are not considered by the superiors in decision making, and they may influence the perception that the work of the colleague in the service is unsatisfactory and generate the sense of lack of compliance with professional ethics and the impression of disloyalty by the colleagues. This way, it is important to value the professionals in order to provide excellence in the services rendered, also highlighting the benefits of an environment favorable to the good routine of work and, later, to workers' quality of life.

Keywords: Occupational health; professional burnout; fatigue.

\footnotetext{
${ }^{1}$ Mestrando do Programa de Inovação e Tecnologia em Enfermagem na USP (EERP) - Faculdade Presidente Antônio Carlos de Porteirinha, Faculdade Verde Norte (FAVENORTE). Brasil. E-mail: ernandesgdias@yahoo.com.br.

2 Graduanda em Enfermagem - Faculdade Presidente Antônio Carlos de Porteirinha, Brasil. E-mail: malufaria@live.com.
} 


\section{INTRODUÇÃO}

De acordo Witter e Paschoal (2010), o estresse pode ser entendido como uma reação psicofisiológica complexa do organismo em resposta a algo que ameace sua homeostase. Diante disso, entende-se que o estresse surge em resposta a algo que cause o desequilíbrio nas dimensões fisiológicas e psicológicas.

A palavra "stress", do inglês, deu origem ao termo estresse em português, que, de acordo com o Dicionário Aurélio da Língua Portuguesa (AURÉLIO, 2016), significa a junção de perturbações orgânicas e psíquicas desencadeadas por estímulos e/ou agentes agressores presentes no ambiente ou por estado emocional.

Como estressores do ambiente físico, pode-se citar o ruído, iluminação, temperatura, higiene, intoxicação, clima, ergonomia, entre outros. E, como principais demandas estressantes, têm-se: o trabalho em turnos, trabalho noturno, sobrecarga de trabalho, exposição a riscos e perigos no ambiente de trabalho. (SEGANTIN; MAIA, 2007). As consequências do "stress" incidem nas instituições organizacionais e põe em situação de risco a motivação, o desempenho, a produtividade, a autoestima e a saúde de seus membros.

Diante dessa premissa, torna-se considerável aludir que o trabalho compreende um papel crucial na vida das pessoas e é um fator notável na formação da identidade e na inserção social desses indivíduos à vista disso, considera-se que o bem-estar adquirido pelo equilíbrio entre as expectativas, em relação à atividade profissional e à sua concretização é um dos fatores que constituem a qualidade de vida. (ABREU et al., 2002). Isso posto, pode-se depreender que essa qualidade de vida se dá por meio da satisfação nos determinantes renda, emprego, objetos possuídos e qualidade de habitação, segurança, privacidade e afeto, bem como motivação, relações de autoestima, apoio e reconhecimento social.

Desse modo, infere-se a relação entre o estresse e o trabalho, uma vez que o estresse ocupacional pode ser conceituado como consequência das relações complexas que se processam entre condições de trabalho, condições externas ao trabalho e características individuais do trabalhador, nas quais as demandas do trabalho excedem as habilidades do trabalhador para enfrentá-las. (FERNANDES; MEDEIROS; RIBEIRO, 2008).

Nesse contexto, o estresse ocupacional refere-se aos estímulos do ambiente de trabalho que exigem respostas adaptativas por parte do trabalhador e que excedem sua habilidade de enfrentamento; esses estímulos são chamados de estressores organizacionais e se manifestam de alguma maneira no exterior do organismo humano. (GENUÍNO; GOMES; MORAES, 2010).

Desse modo, ressalta-se que devido à sua relevância, no entendimento do tema estresse ocupacional, os estressores ocupacionais têm sido pesquisados em muitas profissões, como professores, profissionais de saúde, policiais militares e gestores. No 
que diz respeito ao campo da prestação de serviços relacionados ao comércio, percebese que ainda há uma carência de pesquisas sobre o estresse ocupacional. Nesse contexto, uma pesquisa sobre o estresse ocupacional é oportuna e relevante.

Para atender a demanda e atingir metas por exigência de um mercado competitivo, as organizações necessitam, cada vez mais, administrar mudanças em seu ambiente organizacional, como carga horária excessiva, ambiente psicologicamente insatisfatório, aumento na velocidade de produção, dentre outros fatores que contribuem para o surgimento do estresse ocupacional.

$\mathrm{Na}$ tentativa de solucionar a problemática de quais fatores se relacionam ao estresse ocupacional nos trabalhadores de uma autoescola em Janaúba-MG, objetivouse, por meio deste estudo, traçar o perfil sociodemográfico e histórico ocupacional dos trabalhadores, assim como, identificar os principais fatores estressores entre os colaboradores da Autoescola.

\section{METODOLOGIA}

Trata-se de um estudo descritivo de natureza quantitativa, realizado na empresa de nome fantasia Autoescola Flor de Laranjeira, cuja principal atividade econômica é a prestação de serviços no ramo de autoescola, na cidade de Janaúba, norte de Minas Gerais. Adotou-se um nome fictício para a Autoescola, a fim de preservar a identidade da instituição e dos profissionais envolvidos.

A população do estudo compreendeu 18 funcionários que atuam nos diferentes setores da empresa. Na seleção dos participantes, houve a perda de dois sujeitos, um por estar em férias e outro que cobria as férias de um profissional, há menos de 30 dias no posto de trabalho. Em consideração aos preceitos éticos que envolvem a pesquisa com seres humanos, estabelecidos pela Resolução 466/2012 (BRASIL, 2012), todos os participantes elegíveis para a pesquisa assinaram o Termo de Consentimento Livre e Esclarecido, autorizando o uso das informações cedidas na fase de coleta de dados. Ressalta-se que o projeto de pesquisa para este estudo foi avaliado e aprovado pelo Comitê de Ética em Pesquisa da Universidade Presidente Antônio Carlos (UNIPAC) sob o Parecer Consubstanciado número 1.445.551.

Os dados foram coletados no período de maio a junho de 2016 , no próprio local de trabalho, em horário de serviço, por meio da aplicação de um questionário elaborado pelos pesquisadores, o qual buscou identificar e investigar os fatores que estão relacionados ao estresse nos funcionários da empresa.

O instrumento de coleta de dados foi subdivido em três dimensões: Parte I, referente às informações pessoais (dados socioeconômicos), Parte II, referente às informações ocupacionais, e Parte III, referente às informações específicas causadoras de estresse. 
$\mathrm{Na}$ análise, os dados foram tabulados e apresentados em forma de tabelas e gráficos e foram tratados por meio do método estatístico de percentagem e comparados com a literatura que trabalha essa temática. Por se tratar de valores que representam quantidade de seres humanos, optou-se pelo arredondamento dos valores percentuais.

\section{RESULTADOS}

\subsection{Dados sociodemográficos, tempo e carga horária de trabalho}

Os profissionais participantes da pesquisa representaram $90 \%$ da população total. Nessa perspectiva, participaram do estudo profissionais do sexo masculino (72\%) e feminino (28\%). A idade variou de 25 a 45 anos e o estado civil, entre casados e solteiros. Quanto à escolaridade, $78 \%$ têm $2^{\circ}$ Grau completo (Tabela 1 ).

Tabela 1. Distribuição dos participantes segundo dados sociodemográficos entre trabalhadores da Autoescola Flor de Laranjeira. Janaúba-MG. 2016.

\begin{tabular}{llll}
\hline Variável & & N & $\%$ \\
\hline Sexo & Masculino & 13 & 72 \\
& Feminino & 05 & 28 \\
Faixa Etária & Até 25 & 05 & 28 \\
(anos) & 26 a 30 & 06 & 33 \\
& 31 a 35 & 02 & 11 \\
& 36 a 45 & 04 & 22 \\
& 46 a 56 & 01 & 06 \\
Estado Civil & Solteiro & 09 & 50 \\
& Casado & 09 & 50 \\
Escolaridade & Ensino Médio Completo & 14 & 78 \\
& Ensino Superior & 04 & 22 \\
\hline
\end{tabular}

Fonte: Elaborada pelos autores a partir dos dados da pesquisa. 2016.

Quanto ao tempo de trabalho nessa empresa, observa-se que $50 \%$ dos profissionais têm um tempo de 2 a 8 anos trabalhados. $O$ tempo de atuação no mercado de trabalho, nesse ramo de serviço, é de mais de 10 anos para 10 trabalhadores, sendo que 11 cumprem carga horária de trabalho semanal superior a 40 horas (Gráfico 01).

\subsection{Dados relacionados diretamente ao trabalho}

A Tabela 2 mostra os dados relacionados diretamente ao trabalho que podem desencadear o estresse dentro da ocupação de cada profissional. Diante dos dados dessa tabela, percebe-se que $28 \%$ dos profissionais levam serviços da empresa para serem resolvidos em suas casas. Os profissionais que responderam positivamente a esta questão foram aqueles que desempenham funções de coordenação, gerência e liderança. Entre os participantes, 33\% sentem-se sobrecarregados com as atividades que desempenham na empresa. Além disso, 50\% não encontram reconhecimento pela atividade que exercem na empresa, por parte da gerência. 
Figura 1. Tempo e carga horária de trabalho.

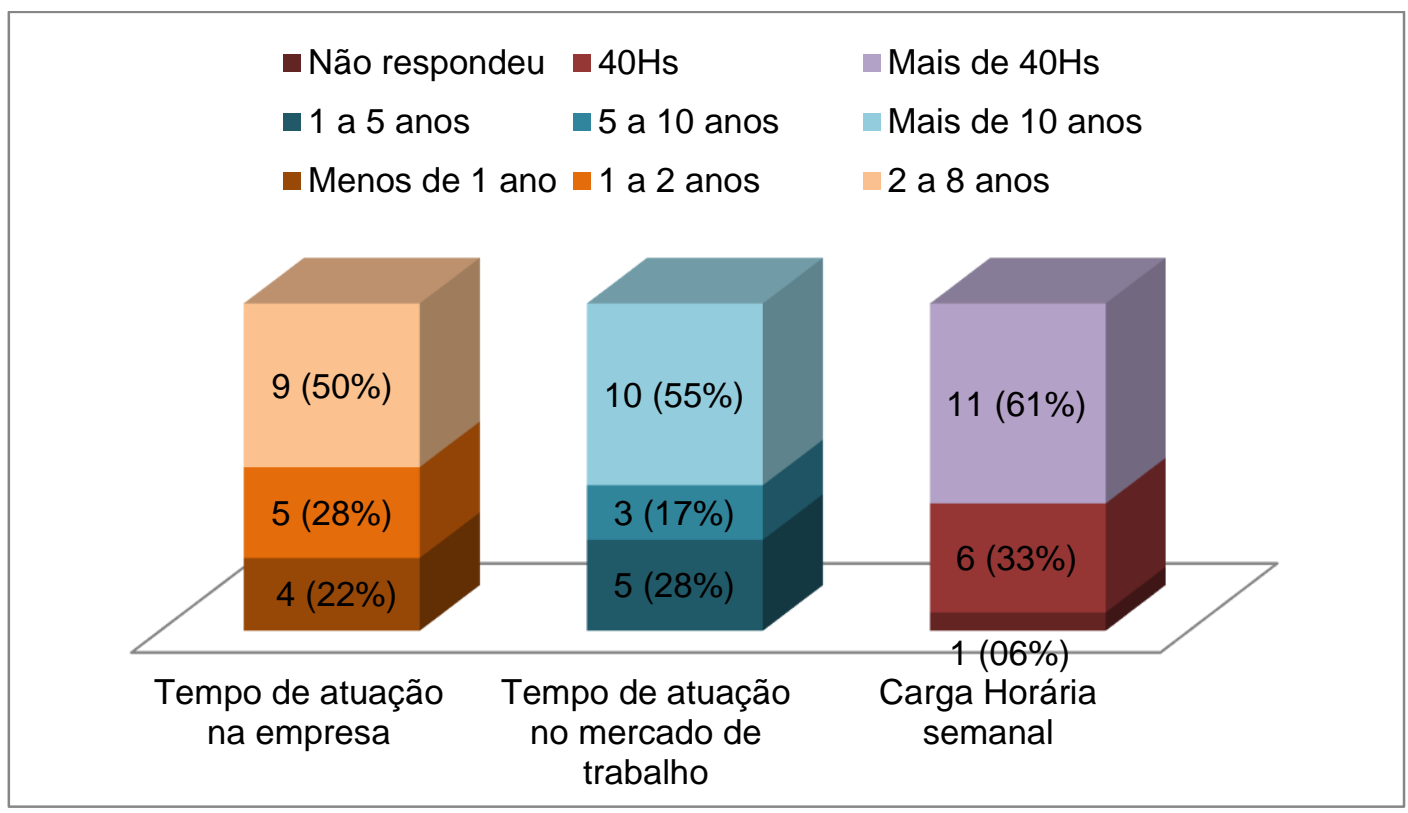

Fonte: Elaborada pelos autores a partir dos dados da pesquisa. 2016.

Outro fator importante é a liberdade em desenvolver as atividades profissionais na empresa, $28 \%$ dos pesquisados afirmaram ter essa limitação. Ressalta-se que $55 \%$ desses consideram que as responsabilidades de seu trabalho são insuficientemente definidas. Além disso, $28 \%$ deles afirmam que as atividades que desempenham são interrompidas com frequência por serem solicitados que cumpram outras funções. Ademais, $55 \%$ dos profissionais consideram que seu trabalho é controlado de forma excessiva pela empresa.

Além dessas informações relacionadas diretamente ao trabalho e que podem causar o estresse, tem-se $45 \%$ de profissionais que afirmam que suas sugestões e recomendações não são levadas em consideração pelos seus superiores. Observa-se, ainda, que $17 \%$ dos pesquisados dificilmente conseguem apoio ou recursos para 0 aprimoramento de sua atuação profissional.

As divergências vivenciadas no trabalho atrapalham, muitas vezes, as responsabilidades familiares e pessoais para $22 \%$ dos pesquisados. Por outro lado, também $22 \%$ dos profissionais consideram que suas atividades profissionais ficam prejudicadas devido ao excessivo número de clientes que atendem. Ressalta-se que $11 \%$ dos profissionais se sentem tensos ao prestar atendimento ao cliente.

Em relação aos relacionamentos dentro da empresa, observa-se que $50 \%$ dos profissionais afirmam que há falta de lealdade e cooperação entre os colegas de trabalho. Além disso, $50 \%$ também afirmam que a ética profissional é ignorada por muitos colegas na instituição. Percebe-se que $45 \%$ dos profissionais percebem a formação de grupos e "panelinhas" que dominam o ambiente de trabalho. Quanto ao desempenho dos colegas de trabalho, $78 \%$ dos profissionais consideram que é 
insatisfatório. Apesar das informações anteriores, não há dificuldade de relacionamento entre os colegas de trabalho.

Tabela 2. Fatores diretamente relacionados ao trabalho que podem desencadear o estresse nos trabalhadores da Autoescola Flor de Laranjeira. Janaúba-MG. 2016.

\begin{tabular}{|c|c|c|c|c|}
\hline \multirow{2}{*}{ Variável } & \multicolumn{2}{|c|}{ Sim } & \multicolumn{2}{|c|}{ Não } \\
\hline & $\mathrm{N}$ & $\%$ & $\mathrm{~N}$ & $\%$ \\
\hline $\begin{array}{l}\text { Costuma levar serviço para casa ou dificuldade de se desligar do } \\
\text { trabalho. }\end{array}$ & 05 & 28 & 13 & 72 \\
\hline Sente-se sobrecarregado com o seu trabalho & 06 & 33 & 12 & 67 \\
\hline Sente que seu trabalho e esforço não são reconhecidos & 09 & 50 & 09 & 50 \\
\hline Atividades frequentemente interrompidas & 05 & 28 & 13 & 72 \\
\hline Trabalho controlado pela gerencia & 10 & 55 & 08 & 45 \\
\hline Sugestões não são levadas em consideração & 08 & 45 & 10 & 55 \\
\hline Tem dificuldade de se relacionar com os colegas de trabalho & - & - & 18 & 100 \\
\hline Falta de lealdade e cooperação entre os colegas & 09 & 50 & 09 & 50 \\
\hline Ética profissional é ignorada pelos colegas de trabalho & 10 & 55 & 08 & 45 \\
\hline Existem "panelinhas" no meio de trabalho & 08 & 45 & 10 & 55 \\
\hline Desempenho profissional de certos colegas é insatisfatório & 14 & 78 & 04 & 22 \\
\hline $\begin{array}{l}\text { Divergências no trabalho atrapalham responsabilidades pessoais } \\
\text { e familiares }\end{array}$ & 04 & 22 & 14 & 78 \\
\hline $\begin{array}{l}\text { Atividades profissionais prejudicadas pelo excessivo número de } \\
\text { clientes }\end{array}$ & 04 & 22 & 14 & 78 \\
\hline Sinto-me tenso ao prestar atendimento ao cliente & 02 & 11 & 16 & 89 \\
\hline Não possuo apoio e recursos para aprimoramento profissional & 03 & 17 & 15 & 83 \\
\hline
\end{tabular}

Fonte: Elaborada pelos autores a partir dos dados da pesquisa. 2016.

\subsection{Dados relacionados ao ambiente de trabalho}

Os fatores que estão relacionados ao ambiente de trabalho se encontram descritos no Gráfico 2. A saber, $28 \%$ dos pesquisados afirmam que a instituição não tem interesse em propiciar as condições necessárias para o desenvolvimento adequado das atividades desempenhadas. Observa-se que $39 \%$ dos pesquisados afirmam que a empresa não dispõe de recursos e instalações apropriadas para cumprir com as responsabilidades profissionais dos trabalhadores. Nessa perspectiva, 6\% dos pesquisados afirmam perceber o ambiente de trabalho como desagradável. 
Figura 2. Dados relacionados ao ambiente de trabalho que podem desencadear estresse ocupacional. Janaúba-MG. 2016.

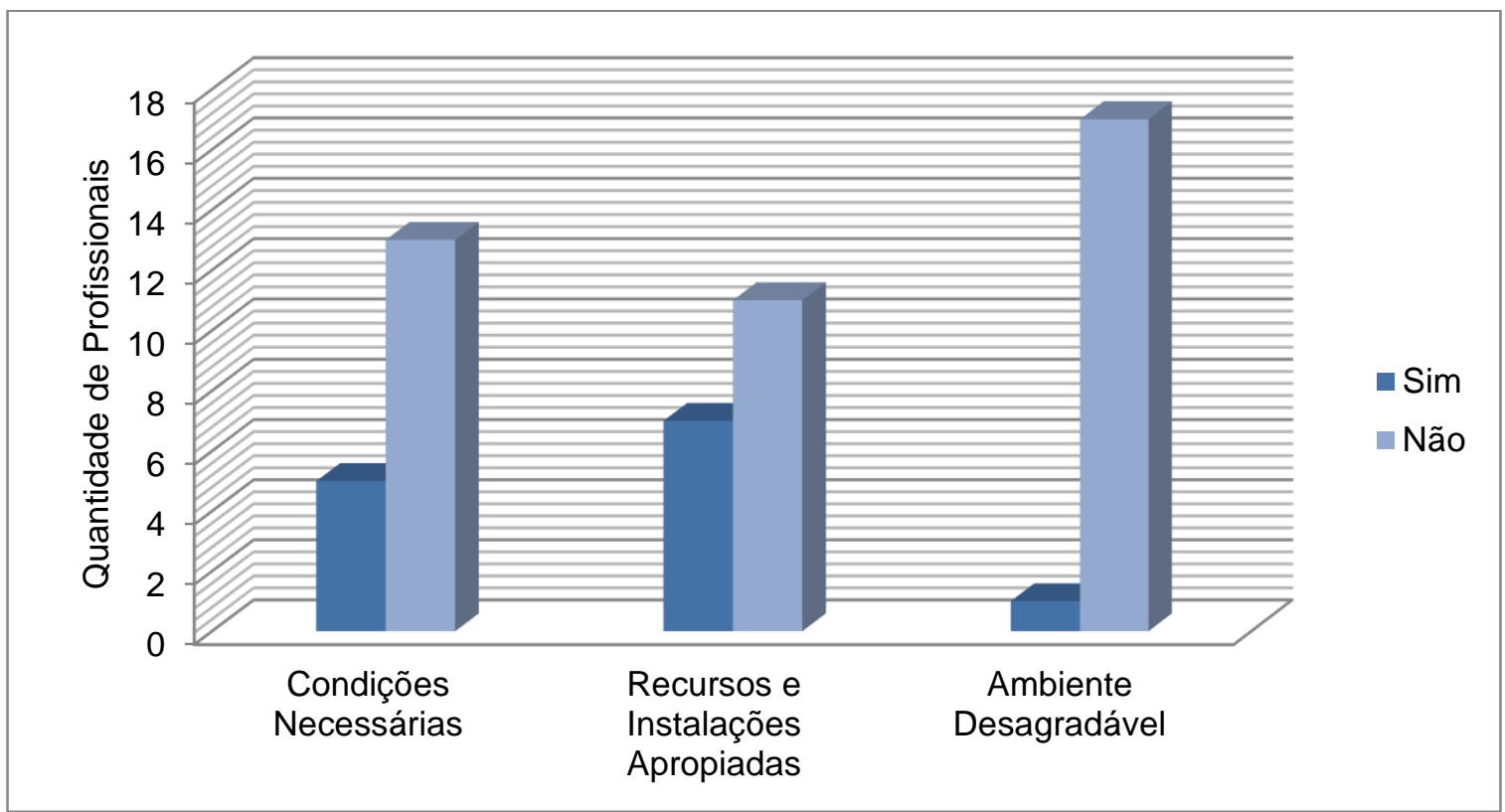

Fonte: Elaborada pelos autores a partir dos dados da pesquisa. 2016.

\section{DISCUSSÃO}

O trabalho é uma das fontes de satisfação humana, como autorrealização, manutenção de relações interpessoais e sobrevivência, por outro lado, pode ser fonte de adoecimento quando existem fatores de risco para a saúde e o trabalhador não dispõe de suporte físico e mental suficiente para se proteger dos perigos a que é exposto.

A maioria dos profissionais pesquisados são do sexo masculino. Nesse sentido, o Instituto Brasileiro de Geografia e Estatística (IBGE, 2012), em 2011 aponta que a distribuição da população ocupada no comércio era de $19,6 \%$ do sexo masculino e $17,5 \%$ do sexo feminino. Além de que a profissão de "lecionar direção em autoescola" pode ser vista pela sociedade como uma tarefa masculina.

Ressalta-se, ainda, a respeito da carga horária semanal trabalhada, que, mesmo que neste estudo a carga horária não se tenha correlacionado diretamente com o estresse, ela pode provocar fadiga e influenciar na ocorrência de erros, interferindo na qualidade do serviço. Portanto, a carga horária apresenta relevância no contexto da saúde do trabalhador. (MANARA, 2014). Além disso, a carga horária elevada está entre as principais causas de acidentes de trabalho, pois causa exaustão e perda de concentração. (SANTOS; FRAZÃO; FERREIRA, 2011).

O acúmulo de tarefas sobrecarrega os profissionais, sendo um desafio entre o que há para ser feito e o tempo disponível para fazê-lo, deixando o trabalhador submetido à enorme tensão, particularmente, diante da responsabilidade de efetuar um trabalho 
qualificado, aumentando o cansaço, o desgaste e o esgotamento crônico, podendo causar o estresse. (VALERETTO; ALVES, 2013; FELLI; BAPTISTA, 2015).

Tratando-se dos relacionamentos interpessoais, ressalta-se que a principal classe estressora são aquelas que se referem a outras pessoas. (MARTINS et al. 2014). A dificuldade de relacionamento gera tensões e conflitos, os membros perdem o espaço para o compartilhamento de experiências no ambiente de trabalho e a oportunidade de contribuir com ideias, minando a força da equipe, que implica em dificuldades no processo do trabalho, afetando a qualidade no trabalho. (VALERETTO; ALVES, 2013).

As condições sob as quais o trabalho é desenvolvido também são consideradas um fator que pode levar ao adoecimento. Condições de trabalho envolvem o ambiente onde este é realizado, materiais utilizados entre outros. (BAIÃO; CUNHA, 2013).

Vale ressaltar, ainda, que a falta de recursos, tanto humano como materiais, constitui um fator estressante importante, pois pode limitar a atuação dos profissionais, influindo diretamente na qualidade do serviço prestado. Pode-se perceber, também, que muitos profissionais não se permitem buscar estratégias para ultrapassar os obstáculos, podendo gerar conflitos, insatisfações, estresse, angústias que desencadeiam sentimentos de sofrimento no trabalho. (VALERETTO; ALVES; 2013).

Dessa forma, é importante que os gestores somem esforços junto aos profissionais para amenizar as situações estressantes presentes no ambiente de trabalho, uma vez que estas têm relação estreita com o sofrimento e o desenvolvimento de doenças ocupacionais.

\section{CONCLUSÃO}

Conclui-se que os principais e mais frequentes fatores desencadeadores do estresse ocupacional foram o excessivo controle por parte da gerência nas atividades desempenhadas, responsabilidade não claramente definida e falta de reconhecimento pelo trabalho realizado. Acredita-se que esses fatores podem justificar que os trabalhadores entendam que suas sugestões não são consideradas pelos superiores, na tomada de decisões, assim como podem influenciar na percepção de que o trabalho do colega de serviço seja insatisfatório e gerar a sensação de falta de cumprimento da ética profissional e impressão de deslealdade por parte dos colegas.

Apesar das evidencias, os profissionais tendem a tolerar as situações estressoras e mantêm boas relações dentro do ambiente de trabalho. Nesse sentido, é importante que as instituições criem espaços onde os profissionais possam se expressar e debaterem esse tipo de situações que possam, ao longo do tempo, provocar adoecimento.

Dessa forma, é importante a valorização dos profissionais, de modo a proporcionar excelência em seus serviços prestados, destacando, ainda, os benefícios 
de um ambiente favorável à boa rotina de trabalho e, posteriormente, à qualidade de vida dos trabalhadores.

\section{REFERÊNCIAS}

ABREU, K. L. et al. Estresse ocupacional e Síndrome de Burnout no exercício profissional da psicologia. Psicologia: ciência e profissão, v. 22, n. 2, p. 22-29, 2002. Disponível em: <http://dx.doi.org/10.1590/S1414-98932002000200004>. Acesso em: 16 ago. 2016.

AURÉLIO. Dicionário do Aurélio Online. Dicionário de Português. (2008 - 2016). Disponível em: <https://dicionariodoaurelio.com/estresse>. Acesso em: 08 nov. 2016.

BAIÃO, R. G. C.; CUNHA, L. P. Doenças e/ou disfunções ocupacionais no meio docente: uma revisão de literatura. Revista Formação@Docente, v. 5, n. 1, p. 6-21, 2013.

BRASIL. Ministério da Saúde. Conselho Nacional de Saúde. Comissão Nacional de Ética em Pesquisa. RESOLUÇÃO №196/96/466/2012 versão 2012. Disponível em: <http://conselho.saude.gov.br/web_comissoes/conep/index.html>. Acesso em: 09 dez. 2016.

FELLI, V. E. A. et al. Sistema de Monitoramento da Saúde do trabalhador de Enfermagem. Saúde do trabalhador de enfermagem. Barueri, SP: Manole, p. 204254, 2015.

FERNANDES, S. M. B. A.; MEDEIROS, S. M.; RIBEIRO, L. M. Estresse ocupacional e o mundo do trabalho atual: repercussões na vida cotidiana das enfermeiras. Revista Eletrônica de Enfermagem. v. 10, n, 2, p. 414-427. 2008. Disponível em: <https://www.fen.ufg.br/revista/v10/n2/v10n2a13.htm>. Acesso em: 12 out. 2016.

GENUÍNO, S. L. V. P.; GOMES, M. S.; MORAES, E. M. O Estresse Ocupacional e a Síndrome de Burnout no Ambiente de Trabalho: Suas Influências no Comportamento dos Professores da Rede Privada do Ensino Médio de João Pessoa. Revista

Anagrama: Revista Científica Interdisciplinar da Graduação. v. 3, n. 2, p. 1-9, 2009.

INSTITUTO BRASILEIRO DE GEOGRAFIA E ESTATÍSTICA - IBGE. Mulher no mercado de trabalho: perguntas e respostas. 2012. Disponível em:

<http://www.ibge.gov.br/home/estatistica/indicadores/trabalhoerendimento/pme_nova/ Mulher_Mercado_Trabalho_Perg_Resp_2012.pdf>. Acesso em: 21 dez. 2015.

MANARA, A. Estudo relaciona carga horária de trabalho com estresse de enfermeiros. 2014. Disponível em: <http://200.144.182.150/aun/exibir?id=6416>. Acesso em: 13 jan.2016.

MARTINS, L. F. et al. Esgotamento entre profissionais da Atenção Primária à Saúde. Ciência \& Saúde Coletiva, v. 19, n. 12, p. 4939-4750, 2014. Disponível em: <http://dx.doi.org/10.1590/1413-812320141912.03202013>. Acesso em: 23 nov. 2016.

SANTOS T. M. B.; FRAZÃO, I. S.; FERREIRA, D. M. A. Estresse ocupacional em enfermeiros de um hospital universitário. Cogitare Enfermagem. v. 16, n. 01, p. 76-81, 2011. 
SEGANTIN, B. G. O.; MAIA, E. M. F. L. Estresse vivenciado pelos profissionais que trabalham na saúde. 2007. Monografia (Conclusão de Curso de Especialização em Saúde da Família). Instituto de Ensino Superior de Londrina - INESUL. Londrina.

VALERETTO, F. A.; ALVES, D. F. Fatores desencadeantes do estresse ocupacional e da síndrome de burnout em enfermeiros. Revista Saúde Física \& Mental, v. 3, n. 2, p. $1-11,2014$.

WITTER, G. P.; PASCHOAL, G. A. Estresse profissional na Base SciELO. Brazilian Journal of Health. v. 1, n. 3, p. 171-185, 2010. 